\title{
Melittin Regulates Iron Homeostasis as a Key Mediator of Macrophage Polarization in Rat Lumbar Spinal Stenosis
}

Hyunseong Kim

Jaseng Medical Foundation

Jin Young Hong

Jaseng Medical Foundation

Wan-Jin Jeon

Jaseng Medical Foundation

Junseon Lee

Jaseng Medical Foundation

Yoon Jae Lee

Jaseng Medical Foundation

In-Hyuk Ha ( $\sim$ hanihata@gmail.com )

Jaseng Medical Foundation https://orcid.org/0000-0002-5020-6723

Research article

Keywords: melittin, lumbar spinal stenosis, M1 macrophages, M2 macrophages, iron homeostasis, iron metabolism, iron deposition

Posted Date: December 29th, 2021

DOI: https://doi.org/10.21203/rs.3.rs-1194869/v1

License: (c) (1) This work is licensed under a Creative Commons Attribution 4.0 International License.

Read Full License 


\section{Abstract}

\section{Background}

Lumbar spinal stenosis (LSS) is defined as the narrowing of the spinal canal, which compresses the nerves traveling through the lower back into the legs. Inflammation is the most common cause of LSS. Chronic pain induced by nerve damage results from chronic inflammation, and the inflammation response worsens with elevated iron stores. Furthermore, macrophage polarization to the M1 (inflammatory) or M2 (anti-inflammatory) type is essential for controlling host defense or repairing tissues. However, the precise function of macrophage polarization in iron release or retention in LSS pathophysiology is not well-understood. Here, we introduce melittin to modulate macrophage polarization related to iron metabolism for LSS treatment.

Methods

Primary peritoneal macrophage were cultured in 200 or $500 \mathrm{ng} / \mathrm{mL}$ of melittin and $\mathrm{FeSO}_{4}$-containing medium for $24 \mathrm{~h}$. Macrophage polarization was assessed by Immunofluorescence staining to CD86 or Arg1 antibodies. In an in vivo rat model of LSS, melittin were administered at 100 and $250 \mu \mathrm{g} / \mathrm{kg}$, and in vivo effects of melittin on iron deposition-induced macrophage polarization was evaluated by immunochemistry, real time-PCR, western blot, and flow-cytometry. The locomotor functions were assessed by BBB, ladder scoring, and Von Frey test for up to 3 weeks.

Results

In vitro experiments demonstrated that macrophages can be polarized toward an $\mathrm{M} 2$ phenotype after melittin treatment in iron-insulted primary macrophages. Treatment with 100 and $250 \mu \mathrm{g} / \mathrm{kg}$ melittin in a rat LSS model increased the proportion of M2 macrophages in the damaged spinal cord. Moreover, we found that melittin attenuated iron overload-induced M1 polarization via regulating iron metabolismrelated genes in LSS rats. As a result, melittin improved locomotor recovery and stimulated axonal growth following LSS.

Conclusions

Melittin can promote functional recovery in LSS models by activating M2 macrophages via controlling macrophage iron metabolism, suggesting the potential applications of melittin for treating LSS.

\section{Background}

Low back pain, paresthesia in the legs, muscle weakness, numbness, and intermittent claudication are common symptoms of lumbar diseases in the elderly population [1]. With the increasing average life expectancy in modern society, the number of patients with degenerative diseases continues to rise [2]. Lumbar spinal stenosis (LSS), a representative degenerative lumbar spinal condition causing low back pain, is a narrowing of the central spinal canal or nerve root canal. Compression of the nerves traveling 
through the lower back into the legs leads to lower back pain and complex neurologic symptoms $[3,4]$. There are various reasons for the narrowing of the spinal canal, with hypertrophy of the lumbar facet joints or ligamentum flava being a major cause $[5,6]$. The ligamentum flavum is a ligament that supports the facet joints and is composed of fibroblasts and extracellular matrix. Degenerative changes trigger inflammation and fibrosis, and the hardened and thickened ligamentum flava compress the nerves [7]. Inflammation is a major factor that induces hypertrophy of the ligamentum flava, during which proinflammatory factors are released around the compressed nerves to exacerbate symptoms [8-10]. Particularly, LSS is a slow-progressing degenerative process compared to other conditions such as herniated disc. Ameliorating inflammation caused by the narrowing of the spinal canal to reduce pain has been proposed as a basic treatment for the condition rather than restoring the spinal canal to the original state $[11,12]$. Therefore, as discussed in numerous previous studies, inflammation is central to the mechanism that causes pain and degeneration of the lumbar spine. However, few studies have focused on how inflammatory mediators contribute to lower back pain in LSS.

According to a recent study, macrophages play a critical role in the initiation, maintenance, and resolution of inflammation, and excessive iron deposition induces inflammatory responses through M1 macrophage polarization [13]. In addition, the iron accumulated M1 macrophages in the damaged spinal cord tissues show increased expression of the inflammatory cytokine tumor necrosis factor [14].

High iron levels and iron-related virulence factors have been reported as risk factors for neurodegenerative disorders, including Alzheimer's disease, Parkinson's disease, and multiple system atrophy [16], indicating that regulation of iron levels in macrophages is an effective strategy for fundamental control of inflammation. Herein, we introduce the melittin to modulate the iron metabolism in macrophage polarization after LSS.

Bee venom is a complex substance that has been used clinically as a medicinal acupuncture for the treatment of intervertebral disc disease. Melittin, the major peptide component constituting $50 \%$ of bee venom, binds to various types of cells. It has anti-inflammatory and antibacterial, strong analgesic, and immune system-boosting effects $[17,18]$. Particularly, melittin has been known to induce antiinflammatory effects by preferentially binding to $M 2$ macrophages $[19,20]$. Therefore, we hypothesized that melittin could control macrophage polarization by regulating the homeostasis of iron metabolism in macrophages in LSS and evaluated its potential therapeutic effects in terms of pain relief and functional recovery.

\section{Methods}

In vitro cultures for peritoneal macrophages

Peritoneal macrophages were obtained from male Sprague-Dawley (SD) rats (10-12 weeks old; Daehan Bio Link, Chungju, Korea). All procedures were approved by the Jaseng Animal Care and Use Committee (JSR-2019-09-022-001). Rats were housed in standard cages at a constantly controlled temperature (23$25^{\circ} \mathrm{C}$ ) and humidity (45-50\%) with a 12-h light/dark cycle. All animals had free access to food and water. 
Rats were injected intraperitoneally with $10 \mathrm{ml}$ of $3 \%(\mathrm{w} / \mathrm{v})$ thioglycollate (Becton Dickinson, Sunnyvale, CA, USA). Rats were euthanized from 4 days after injection and their peritoneal cavity was opened. The peritoneal fluid was harvested from the peritoneal cavity by rinsing $40 \mathrm{~mL}$ of RPMI-1640 (Hyclone, South Logan, UT, USA), and then centrifuged at $4^{\circ} \mathrm{C}$ for $3 \mathrm{~min}$ at 1,500 $\mathrm{rpm}$. The supernatant was discarded, and cell pellets were resuspended in $1 \mathrm{~mL}$ of red blood cell lysis buffer (Invitrogen, Carlsbad, CA, USA) at room temperature for $5 \mathrm{~min}$ and then washed in phosphate-buffered saline (PBS; Gibco, Grand Island, NY, USA). Macrophages were resuspended in RPMI-1640 medium supplemented with 10\% fetal bovine serum (Gibco) and $1 \%$ penicillin-streptomycin (Gibco), and then seeded at $1 \times 10^{6}$ cells per well onto poly-dlysine coated 12-mm glass coverslips (Paul Marienfeld GmbH and Co., Lauda-Königshofen, Germany) in 24-well plates for immunocytochemistry.

Iron(II)sulfate heptahydrate $\left(\mathrm{FeSO}_{4}\right)$ and melittin treatment

Iron-mediated macrophage polarization was performed in an in vitro setting as described previously [21]. Briefly, primary macrophages were cultured in a poly-d-lysin coated plates for $24 \mathrm{~h}$ after cell seeding. For $\mathrm{FeSO}_{4}$ (Sigma-Aldrich, St. Louis, MO, USA) treatment after $24 \mathrm{~h}$, an iron stock solution was prepared using $278 \mathrm{mg}$ of $\mathrm{FeSO}_{4}$ dissolved in $10 \mathrm{~mL}$ PBS. After filtering using a $0.2 \mu \mathrm{m}$ membrane, $\mathrm{FeSO}_{4}$ was added to the cell culture media at a final concentration of $50 \mu \mathrm{M}$ for $30 \mathrm{~min}$. After $30 \mathrm{~min}$ of incubation at $37^{\circ} \mathrm{C}$ in a $\mathrm{CO}_{2}$ incubator, melittin was added to the $\mathrm{FeSO}_{4}$-containing medium at a concentration of 200 or $500 \mathrm{ng} / \mathrm{mL}$. The cells were further incubated in $5 \% \mathrm{CO}_{2}$ at $37^{\circ} \mathrm{C}$ for 24 $h$, and samples were used for immunocytochemistry.

\section{Immunocytochemistry}

The macrophages were cultured in 200 or $500 \mathrm{ng} / \mathrm{mL}$ melittin, and the expression of macrophage M1 (CD86) and M2 (Arginase 1; Arg1) markers was detected by immunostaining after $24 \mathrm{~h}$ with five biological replicates per condition. Briefly, the samples were fixed with $4 \%$ paraformaldehyde (PFA; Biosesang, Seongnam, Korea) for $30 \mathrm{~min}$ and washed three times for $5 \mathrm{~min}$ each with PBS. The cells were permeabilized with $0.2 \%$ Triton X-100 in PBS for 5 min, washed two times and blocked with $2 \%$ normal goat serum (NGS) in PBS for $1 \mathrm{~h}$. The following primary antibodies were used: $\operatorname{Arg} 1$ (1:200, Novus, Littleton, CO, USA), CD68 (1:500, Abcam, Cambridge, UK), CD86 (1:100, BD PharMingen, San Diego, CA, USA). The primary antibodies were diluted in $2 \%$ NGS and incubated overnight at $4^{\circ} \mathrm{C}$. After washing with PBS three times for 5 min each, the samples were then incubated for $2 \mathrm{~h}$ with fluorescein isothiocyanate (FITC) or rhodamine-conjugated secondary antibodies (goat anti-rabbit IgG or goat antimouse IgG; Jackson ImmunoResearch Laboratories, West Grove, PA, USA) diluted at 1:300 in 2\% NGS. After $2 \mathrm{~h}$ of incubation at room temperature, the cells were washed three times for 5 min with PBS, mounted with fluorescence mounting medium (Dako Cytomation, Glostrup, Denmark), and images were acquired by confocal microscopy (Eclipse C2 Plus; Nikon, Tokyo, Japan). For quantification of macrophage polarization, five representative images were captured at 200 xmagnification with fixed acquisition parameters and analyzed using counting of ImageJ software. The numbers of $\mathrm{CD}^{+} 6^{+}$or 
$\mathrm{Arg} 1^{+} / \mathrm{ED} 1^{+}$cell was counted and the proportion of $\mathrm{M} 1$ or $\mathrm{M} 2$ double-positive cells was expressed as a percentage of cells that appear bright in response to CD86 or Arg1 by the number of ED $1^{+}$cells.

\section{Rat LSS model and melittin administration}

Male SD rats (7 weeks old, 230-250 g) were obtained from Daehan Bio Link (Chungju, Korea). All procedures were approved by the Jaseng Animal Care and Use Committee (JSR-2018-10-001-A). Rats were housed as described above. The animals were deeply anesthetized with 2-3\% isoflurane gas (Forane; BK Pham, Goyang, Korea), and a dorsal laminectomy was performed at L5 using fine rongeurs. Next, a silicone block $\left(80 \mathrm{kPa}, 4 \times 1 \times 1 \mathrm{~mm}^{3}\right)$ was inserted at the L4 level using no. 5 fine forceps. Shamoperated rats underwent laminectomy only at the $L 5$ level without silicone insertion. The spinal cord was covered with Surgicel ${ }^{\circledR}$ absorbable hemostat (Johnson and Johnson, Arlington, TX, USA) to help achieve hemostasis in the spinal cord. To prevent infection, all rats were injected intramuscularly with $40 \mathrm{mg} / \mathrm{kg}$ cefazolin sodium (Cefazolin ${ }^{\circledR}$, Chong-Kun-Dang Pharm., Seoul, Korea) after suturing. All rats were also administered an oral dose of $10 \mathrm{mg} / \mathrm{kg}$ Children's Tylenol ${ }^{\circledR}$ (Janssen Korea, Inc., Seoul, Korea) after the anesthesia was resolved for pain management. Different concentrations of melittin (100 and $250 \mu \mathrm{g} / \mathrm{kg}$ ) were administered once per day by subcutaneous injection. Injection was started at $30 \mathrm{~min}$ after LSS and continued for 1 or 3 weeks after LSS before sacrifice. The control group was administered the same volume $(400 \mu \mathrm{L})$ of PBS.

\section{Histology}

Sham or LSS rats from each group were deeply anesthetized with 2-3\% isoflurane gas and perfused via cardiovascular system with $0.9 \%$ normal saline (Sigma-Aldrich) and 4\% PFA (Biosesang) for histological staining and immunohistochemistry. The spinal cord at the silicone implantation site was dissected, postfixed overnight in $4 \%$ PFA at $4{ }^{\circ} \mathrm{C}$ and dehydrated with $30 \%$ sucrose in $0.1 \mathrm{M}$ phosphate buffer for 3 days. The samples were cryo-sectioned at $20 \mu \mathrm{m}$ in the sagittal plane. Hematoxylin and eosin (H\&E) staining was performed at the L4 level to evaluate the degree of damage to the spinal cord caused by implantation of the silicone block at 3 weeks. Briefly, the sectioned tissue slides were dipped into hematoxylin for $2 \mathrm{~min}$, washed in running tap water for $2 \mathrm{~min}$, and then stained with eosin for $5 \mathrm{~s}$. The stained sections were dehydrated through a graded series of ethanol, cleared with xylene, mounted with VectaMount ${ }^{\circledR}$ Permanent Mounting Medium (Vector Laboratories, Burlingame, CA, USA), and imaged under an inverted microscope (Nikon, Tokyo, Japan).

\section{Immunohistochemistry}

Immunohistochemistry was performed on spinal cord sections to analyze macrophage subset and painand iron-related markers. Primary antibodies against monocyte/macrophage rabbit anti-CD68 (1:500, Abcam, Cambridge), rabbit anti-TRPV1 (1:100, Alomone, Hadassah Ein Kerem, Israel), guinea pig antiNeuN (1:500, Synaptic Systems, Göttingen, Germany), mouse anti-ferritin heavy chain (1:400, Santa Cruz Biotechnology, Dallas, TX, USA), CD86 (1:100, BD PharMingen), Arg1 (1:200, Novus), and mouse antiNF200 (1:200, Millipore, Billerica, MA, USA) were incubated overnight at $4^{\circ} \mathrm{C}$. The sections were then 
washed three times with PBS and secondary antibodies (fluorescein isothiocyanate-coupled goat antimouse or anti-rabbit or rhodamine-conjugated goat anti-guinea pig, Jackson ImmunoResearch Laboratories, West Grove, PA, USA) were treated at a 1:300 dilution in 2\% normal goat serum. Following incubation at room temperature for $2 \mathrm{~h}$, the sections were washed three times with PBS and mounted with using Dako Mounting Medium (Dako Cytomation). The stained tissue sections were observed by confocal microscopy (Eclipse C2 Plus, Nikon). The fluorescence intensity was analyzed for confocal images captured by the same acquisition settings. Background was subtracted using the subtract background tool in ImageJ software (1.37 v, National Institutes of Health, Bethesda, MD, USA). CD68positive macrophages were manually counted in the silicone implanted area using confocal microscopy images obtained at $100 \times$ magnification. The result was expressed as a percentage or ratio. NF200labeled axons were quantified by using ImageJ. Briefly, NF200-labeled axons were captured at the implantation site under a 100x objective lens of a confocal microscope, and the number of pixels occupied by the NF200 fibers was counted in three images; NF200-positive pixels were divided by the number of pixels in a field.

RNA isolation and real-time polymerase chain reaction ( $q P C R)$

Changes in the mRNA levels of genes related to the inflammation (inducible nitric oxide synthase (iNOS), cyclooxygenase-2 (COX-2), interleukin (IL)-1beta ( $\beta$ ), IL-6, IL-10, tumor necrosis factor-alpha (TNF-a), arginase 1 (Arg1), nuclear factor erythroid 2-related factor 2 (Nrf2)), iron metabolism (ferritin light polypeptide 1, ferritin heavy/light chain (FTH1/FTL), hepcidin antimicrobial peptide (HAMP), divalent metal transporter 1 (DMT1), transferrin receptor (TFRC), ceruloplasmin (CP), and Wingless-type family member 3, 5A (Wnt3, Wnt5a), neurofilament 200 (NF200) were analyzed by qPCR. Total RNA was extracted from the L4 spinal cord using a RNeasy Mini Kit (Qiagen, Hilden, Germany). cDNA was synthesized using oligo dT primers and AccuPower RT PreMix (Bioneer, Daejeon, Korea). Primers were designed using the UCSC Genome Bioinformatics and NCBI databases and are listed in Table 1. Quantitative reverse-transcription PCR (qRT-PCR) was performed in triplicate using iQ SYBR Green Supermix with a CFX Connect Real-Time PCR Detection System (both from Bio-Rad, Hercules, CA, USA). Target gene expression was normalized to that of the $\beta$-actin gene and expressed as a fold-change relative to the control group.

\section{Flow cytometry}

Spinal cord tissues were isolated into single cells for FACS analysis. The tissue under silicone was dissociated using a Neural Tissue Dissociation kit (Miltenyi Biotec, Bergisch Gladbach, NRW, Germany) and gentleMACS Dissociator (Miltenyi Biotec). The dissociated cells were filtered in $20 \mu \mathrm{m}$ cell strainer (FALCON) and centrifuged at 2,000 rpm for $3 \mathrm{~min}$. Flow cytometry was performed to assess cell death following LSS. Apoptotic cell death was detected using a FITC Annexin V Apoptosis Detection Kit I (BD Bioscience, Franklin Lakes, N, USA). Briefly, the cells were collected and stained with $1 \mu \mathrm{L}$ of Annexin Vphycoerythrin and $1 \mu \mathrm{L}$ of propidium iodide in $200 \mu \mathrm{L}$ of $1 \times$ binding buffer and directly analyzed by fluorescence-activated cell sorting (Accuri C6 Plus Flow Cytometer, BD Biosciences). In addition, 
ferroportin expression was evaluated by ferroportin/SLC40A1 antibody (Novus) after fixation with fixation buffer (BD Bioscience). The mean positive cell values, as determined via flow cytometry, were expressed as a percentage relative to the control group.

\section{Enzyme-linked immunosorbent assay}

The expression levels of the pro- and anti-inflammatory markers IL-6 and IL-10 in a separated spinal cord, including at the implantation site, were evaluated by enzyme-linked immunosorbent assay (ELISA). The spinal cord tissues were homogenized in radioimmunoprecipitation assay (RIPA) buffer (GenDEPOT, Barker, TX, USA) containing a proteinase inhibitor (Millipore) using a Taco ${ }^{\mathrm{TM}}$ Prep Bead Beater (GeneReach, Taichung, Taiwan) and centrifuged at 1,000 rpm at $4^{\circ} \mathrm{C}$ for $3 \mathrm{~min}$. Protein concentration was quantified using a bicinchoninic acid (BCA) protein assay kit (Thermo Fisher Scientific, Waltham, MA, USA). The supernatants were examined using ELISA kits (BD Biosciences, Franklin Lakes, NJ, USA) according to the manufacturer's instructions. The final concentrations of cytokines were calculated with respect to the amount of each protein.

\section{Functional assessments}

Locomotor function was assessed for 3 weeks using three tests after LSS. The Von Frey test was used to measure the foot response to pain. The rats were placed in acrylic cages for $15 \mathrm{~min}$ and then stimulated at the test area on the plantar surface. We measured the latency of paw withdrawal in response to mechanical stimulation using a Von Frey filament (Ugo Basile, Varese, Italy). Each value was automatically recorded from three or more measurements and then averaged. The BBB scale was assessed in an open field for 4 min by two independent observers, and the BBB score was calculated from the average of hindlimb movements. The ladder walking test was also performed to confirm the balance and forelimb-hindlimb coordination. All rats walked the runway $(2.5 \mathrm{~cm}$ interval) from left to right three times, and their movements were recorded using a digital camera and calculated as follows: ladder score $(\%)=$ erroneous steps of hind limb/total steps of hind limb $\times 100$.

\section{Statistical analyses}

All numeric data are expressed as the mean \pm standard error of the mean. Comparisons among each group were analyzed using one-way analysis of variance (ANOVA) with Tukey's post-hoc analysis (GraphPad Prism 8, Inc., La Jolla, CA, USA). Differences were considered as statistically significant if the $p$ value

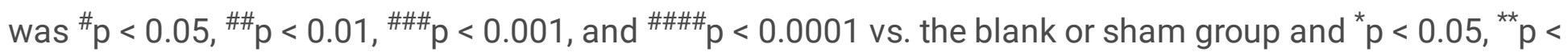
$0.01,{ }^{\star \star *} \mathrm{p}<0.001$, and ${ }^{\star \star \star *} \mathrm{p}<0.0001$ vs. the $\mathrm{FeSO}_{4}$ or control group.

\section{Results}

Melittin promotes a phenotypic switch of $\mathrm{FeSO}_{4}$-induced M1 polarization in primary macrophages 
First, the phenotypic characterization of melittin-treated macrophages was investigated by immunocytochemical staining under in vitro conditions that simulate $\mathrm{FeSO}_{4}$-induced $\mathrm{M} 1$ polarization. The immunocytochemical double staining for $\mathrm{M} 1\left(\mathrm{CD} 86^{+}\right)$or $\mathrm{M} 2\left(\mathrm{Arg}^{+}\right)$markers with macrophages $\left(\mathrm{CD}_{68}{ }^{+}\right)$was carried out (Figure 1A). The population of M1 macrophages expressing CD86 marker was significantly increased by $35.21 \%$ after $\mathrm{FeSO}_{4}$ treatment, while it significantly decreased from $17.05-$ $12.71 \%$ in the melittin groups compared to that in the $\mathrm{FeSO}_{4}$ group, showing a dose-dependent inhibition in its action of inducing M1 polarization (Figure 1B). By contrast, the percentage of $A r g 1^{+} \mathrm{M} 2$ macrophages was shown to increase significantly with increasing melittin dose in $\mathrm{FeSO}_{4}$-treated macrophages (Figure 1C). In addition, macrophages treated only with $500 \mathrm{ng} / \mathrm{mL}$ melittin did significant increase in the percentage of $\mathrm{Arg}^{+} \mathrm{M} 2$ macrophages (Figure S1). Our findings demonstrate that melittin induces a phenotypic switch of macrophage polarization into an M2 anti-inflammatory state.

In vivo administration of melittin to rats with LSS reduces inflammatory response and apoptotic cell death

To investigate the general histologic characteristics of the spinal cord after silicone implantation in the spinal canal, we performed H\&E staining of the sagittal sections at 1 week after LSS. Compression of the spinal cord was observed in the stained sections (Figure S2). Macrophage infiltration into the compressed spinal cord underneath the silicone block was detected by immunohistological staining. CD68-positive cells were not detected in the sham group, whereas CD68-positive cells were densely packed in the spinal cord after LSS and dramatically decreased after melittin administration in a dosedependent manner (Figure 2A). We further analyzed the presence of CD68-positive macrophages at 3 weeks after LSS. Overall, a small population of CD68-positive cells was detected in each group compared to those at 1 week. However, the significant pattern at 3 weeks is similar to that in animals that were administered melittin for 1 week after LSS induction and the number of macrophages positive for CD68 was also shown to decrease significantly in the melittin groups compared to that in the control group (Figure 2B). Changes in IL-6 and IL-10 levels in the spinal cord were determined by ELISA. IL-6, a proinflammatory cytokine, is a key player leading to an inflammatory response and tissue damage in the spinal cord. The level of IL- 6 was significantly higher in the control group than in the sham group at 1 week after LSS and was significantly and dose-dependently decreased after melittin administration in LSS rats (Figure 2C). Additionally, the level of IL-10, an anti-inflammatory cytokine that was significantly lower in the control group than in the sham group at 1 week after LSS, was dose-dependently increased at 1 week after melittin administration in LSS rats (Figure 2D). With regard to cell death at 1 week after melittin administration in LSS rats, we performed further analysis in spinal cord tissue using flow cytometric assay (Figure 2E). The spinal cord cells in early apoptosis were stained with Annexin $\mathrm{V}^{+} / \mathrm{PI}^{-}$, whereas cells in late apoptotic stage were stained with Annexin $\mathrm{V}^{-} / \mathrm{PI}^{+}$. We found that most spinal cord cells at 1 week after LSS were early apoptotic, and a relatively high percentage of Annexin $\mathrm{V}^{+} / \mathrm{PI}^{-}$cells were observed in $25.5 \%$ of the control group compared with that in the sham group. When animals were administered with 100 and $250 \mu \mathrm{g} / \mathrm{kg}$ melittin for 1 week, the apoptotic population of isolated cells from the spinal cord was approximately $14.01 \%$ and $9.6 \%$, respectively (Figure $2 \mathrm{~F}$ ). These findings reveal that 
melittin effectively reduced the inflammatory response and apoptotic cell death in the spinal cord after LSS.

Melittin ameliorates LSS-induced iron accumulation, which was released from activated macrophages in the spinal cord

We next examined whether the CD68-positive macrophage population is linked to impaired iron storage after LSS. Macrophages play key roles in regulating systemic iron homeostasis, which is closely linked to macrophage polarization [22]. Iron overload is indicated by elevated ferritin levels, which is related to the amount of iron stored in cells. At 1 week after LSS, immunohistological staining revealed an increase in the number of ferritin-positive macrophages in the spinal cord after LSS, whereas their population significantly decreased in the melittin group (Figure $3 \mathrm{~A}$ ). The expression levels of iron metabolism related genes, including TFRC, DMT1, ferritin heavy/light chain (FTH1/FTL), CP, HAMP, and ferroportin, were evaluated in the sham, control, and melittin groups at 1 week. qPCR analysis of the spinal cord was performed to confirm the changes in the expression of genes related to iron metabolism at 1 week after melittin administration in LSS rats. TFRC and DMT1 expression was significantly upregulated in the control group compared to that in the sham group and significantly downregulated after melittin administration. These findings demonstrate that LSS induces an increase in iron intake by cells through TFRC and DMT1 and a decrease in iron intake after melittin administration (Figure 3B, C). We also evaluated changes in the expression levels of the iron storage genes FTH1/FTL. FTH1 and FTL expression levels were significantly higher in the control group than in the sham group at 1 week. In contrast to that in the control group, melittin administration to LSS rats significantly downregulated FTH/FTL 1 gene expression (Figure 3D, E). We also investigated changes in the expression of $C P$ and HAMP. CP expression was significantly downregulated in the control group compared to that in the sham group but was upregulated after melittin administration in LSS rats (Figure 3F). Therefore, the upregulation of $C P$ is mainly associated with elevated ferroxidase activity to export iron via $F P N$ after melittin administration in LSS rats. We also confirmed that HAMP expression was significantly increased in the control group. In contrast, HAMP expression was significantly decreased after melittin administration in a dose-dependent manner (Figure 3G). These results demonstrate that elevated HAMP expression can inhibit iron export by binding to FPN and inducing iron deposition within the spinal cord in LSS rats, whereas low expression of HAMP can induce iron efflux to maintain iron homeostasis after melittin administration in LSS rats. We further investigated the iron exporter ferroportin levels in spinal cord cell isolated from tissues of each group using flow cytometry (Figure 3H). FPN positivity dramatically decreased owing to LSS induction, whereas administration of melittin with 100 or $250 \mu \mathrm{g} / \mathrm{kg}$ reversed this, with the positivity significantly increasing in LSS rats compared to that in the control (Figure 3I). Thus, these findings strongly suggest that melittin will be an effective treatment for maintaining iron homeostasis by regulating iron metabolism-related genes after LSS.

Melittin reduces $\mathrm{M} 1$ macrophage activation by suppressing iron accumulation 
Next, we investigated whether intracellular iron deposition induces M1-type macrophage polarization after LSS. M1 macrophages are rich in ferritin and prone to iron accumulation [23]. CD86 is widely used as a marker to identify M1 macrophages. We performed immunohistological staining using CD86 and ferritin antibodies (Figure 4A). CD86/ferritin dual-stained cells were detected at 1 week after LSS. This finding suggests that LSS can induce M1 macrophage polarization with increased iron storage ferritin expression. However, CD86/ferritin dual-stained cells were significantly decreased in the $250 \mu \mathrm{g} / \mathrm{kg}$ melittin group (Figure 4B). Furthermore, qPCR revealed increased gene expression of $i N O S, C O X-2, I L-1 \beta$, $I L-6$, and TNF- $a$ in the spinal cord after LSS at 1 and 3 weeks, whereas the expression of these genes was significantly decreased in the melittin group, which was associated with a reduction in the inflammatory response owing to suppression of M1 macrophages activation (Figure $4 C-G$ ). These findings strongly suggest that melittin administration can reduce M1-type cell polarization by inhibiting iron deposition.

Melittin promotes M2 macrophage polarization and inhibits iron deposition

In our next set of experiments, we confirmed that melittin modulates macrophage polarization toward the M2 phenotype by inhibiting iron deposition. M2 macrophages are characterized by the expression of Arg1, a key enzyme produced by this type of macrophages, and the mannose receptor MRc1 (CD206). We performed immunohistological staining of Arg1 to identify M2-type cell polarization following melittin administration to LSS rats. In addition, we assessed ferritin expression in Arg1-positive M2 macrophages by double staining of ferritin and Arg1 (Figure 5A). Interestingly, Arg1 expression was reduced in the damaged spinal cord at 1 week after LSS. However, ferritin expression was dramatically increased after LSS. In contrast, the number of M2 macrophages positive for Arg1 was significantly increased, whereas ferritin expression was decreased in the melittin groups (Figure 5B). The mRNA expression of Arg 1 was analyzed at 1- and 3-weeks post-operation. The expression level of Arg 1 did not differ among the control and melittin groups but showed significant upregulation at 3 weeks in the melittin groups compared to that in the control group (Figure $5 \mathrm{C}$ ). The representative anti-inflammatory gene $I L-10$ was also significantly upregulated at 1 week in the melittin groups, whereas in 3 weeks after LSS, there was no significant differences between groups (Figure 5D). Therefore, melittin can induce macrophage polarization to an M2 phenotype in LSS rats.

Melittin relieves neuropathic pain by inhibiting TRPV1 in LSS rats

To explore the antinociceptive effects of melittin on TRPV1 expression in dorsal root ganglionic (DRG) sensory neurons, we performed immunohistochemical staining by using TRPV1 from the rat spinal cord at 3 weeks after LSS. TRPV1 is a well-known pain-mediating ion channel expressed in sensory neurons, including DRG neurons, trigeminal ganglionic neurons, and vagal neurons. TRPV1 expression in DRG neurons was increased dramatically after LSS. In contrast, TRPV1 expression in NeuN-positive neurons was dramatically decreased after melittin administration in LSS rats (Figure 6A). Fluorescence colocalization was quantified as the percentage of neurons expressing TRPV1 in NeuN-positive DRG sensory neurons at 3 weeks after LSS. A significant increase in the percentage of TRPV1-NeuN neurons in the DRG was observed in the control group at 3 weeks after LSS (Figure 6B). In contrast, increased TRPV1 
expression was dose-dependently inhibited by melittin administration to LSS rats. Moreover, the mean TRPV1 intensities showed similar expression patterns to those observed in the percentage of TRPV1positive neurons in the DRG (Figure 6C). Therefore, melittin ameliorates neurotrophic pain by downregulating the expression of TRPV1 in the DRG of LSS rats.

Melittin promotes locomotor recovery by preventing axonal loss in LSS rats

We next examined the effect of melittin on axons damaged by implanted silicone by immunohistochemistry of NF200 (an axonal marker) in sagittal spinal cord sections from the implantation site. Axonal damage after LSS appeared to be induced by the implanted silicone. The damaged spinal cord showed improved axonal growth after administration of different concentrations of melittin (Figure 7A). We also quantified the NF200-positive densities and intensities in the spinal cord at the implantation site, which were greater in the melittin groups than in the control group (Figure 7B, C). Furthermore, the changes in several regeneration-related genes, including NF200, Nrf-2, Wnt3, and Wnt5a, were analyzed by qPCR at 1 and 3 weeks after LSS. NF200 gene expression differed between groups at 1 and 3 weeks after LSS (Figure 7D). However, the mRNA level of Nrf-2 in the spinal cord was significantly higher in the $250 \mu \mathrm{g} / \mathrm{kg}$ melittin group than in the control group at 1 week, whereas there was no significant difference between groups at 3 weeks (Figure 7E). The Nrf-2 pathway plays a critical role in protecting against oxidative stress and inducing regeneration of various tissues. In addition, Wnt signaling has been linked to axon regrowth and transport through the Wnt/ $\beta$-catenin pathway (Wnt1 and Wnt3a) or Wnt/PCP pathway (Wnt5a). The expression levels of Wnt3 and Wnt5a were significantly and dose-dependently increased by melittin administration at 1 week (Figure 7F, G). These findings suggest that melittin can promote axonal growth in LSS rats.

Finally, locomotor functions were assessed for up to 3 weeks using three methods (BBB, ladder, and von Frey tests) to determine whether melittin improved functional recovery after LSS. The melittin groups showed increased BBB scores compared to those in the control group until 3 weeks. An average BBB score of 16 points was observed at 3 weeks in the control group. The melittin groups had an average higher BBB score for open field locomotion at 3 weeks after LSS than the control group, with significant differences observed between the melittin groups and control group at 3 weeks; the scores in the melittin groups were approximately two points higher than that in the control group (Figure $7 \mathrm{H}$ ). The horizontal ladder test revealed more noticeable behavioral differences. The control group had a higher foot fault rate of approximately $10 \%$ for 3 weeks than the sham group, whereas the melittin groups showed a decreased foot fault frequency compared to that in the control group at 3 weeks. However, the $250 \mu \mathrm{g} / \mathrm{kg}$ melittin group showed a significant decrease in the foot fault rate for up to 3 weeks (Figure 7I). In addition, we assessed the effect of melittin on mechanical allodynia using the von Frey test. The control group showed an average withdrawal latency of $4 \mathrm{~s}$ for 3 weeks. There was a significant difference between the sham and control groups for up to 3 weeks. In contrast, rats administered different concentrations of melittin showed significantly lower sensitivity than the control group at 3 weeks (Figure 7J). Therefore, melittin improved locomotor functional recovery after LSS in rats by promoting axonal regrowth. 


\section{Discussion}

In this study, we examined the inflammatory responses as the increase in the number of macrophages that penetrated the spinal cord tissues damaged by LSS. Specifically, iron overload was mainly observed in M1 macrophages, whereas iron accumulation was not confirmed in M2 macrophages, which are known to secrete anti-inflammatory cytokines. In addition, administration of melittin, which promotes macrophage transformation from $\mathrm{M} 1$ to $\mathrm{M} 2$, decreased $\mathrm{M} 1$ macrophages and ferritin expression levels. In contrast, increases in M2 macrophages anti-inflammatory factor expression were observed. Moreover, these changes were closely associated with iron metabolism. We found that melittin could regulate iron balance by regulating of TFRC and DMT1, which transport iron into cells. Under the LSS condition, TFRC and DMT1 expression was elevated, and iron was mostly stored in the form of ferritin protein. Originally, to maintain a stable iron homeostasis, CP can be upregulated to increases the oxidation of ferrous iron to ferric iron and facilitate iron export through FPN. Hepcidin, another mediator of iron metabolism, can regulate iron transport by binding to FPN. However, CP was downregulated, and HAMP was upregulated after LSS. Meanwhile, melittin has been shown to be involved in LSS-induced changes in iron metabolism.

Aging involves the deterioration of cell functions and is a major cause of numerous diseases related to failed cellular homeostasis. Various neurodegenerative disorders such as Alzheimer's disease and Parkinson's disease are closely associated with aging, and a decrease in homeostasis due to the accumulation of oxidized proteins and reactive oxygen species (ROS) has been reported [24, 25]. Cells possess an antioxidant system that effectively removes ROS to maintain the balance between oxidants and antioxidants as well as homeostasis [26]. In particular, ROS produced in large quantities by immune cells such as macrophages and neutrophils play an important role in removing foreign substances during infection or inflammatory responses. However, continuous and excessive ROS production and long-term accumulation causes damage to cells and tissues and promotes chronic inflammation and aging [24]. Therefore, failed homeostasis is the underlying cause of oxidative stress and inflammation in degenerative diseases, indicating that homeostasis can be regulated to treat diseases.

Iron is an essential trace metal in metabolism for maintaining homeostasis in humans, and many studies have focused on the relationship between the molecular mechanisms regulating iron metabolism and degenerative disorders related to aging [27]. Iron gradually accumulates in specific cells and tissues during aging and accelerates the decline in mitochondrial functions [28]; iron overload acts as a catalyst in a reaction that produces ROS, thereby damaging tissues and causing diseases.

We confirmed macrophage polarization according to iron levels following LSS and the anti-inflammatory effect and functional recovery through the regulation of macrophage polarization. However, the LSS animal model in this study artificially compresses the nerves when bio-silicone is implanted on the ligamentum flava of the spinal canal, and hypertrophy of the ligamentum flava was not directly induced. Therefore, further studies are needed to investigate the direct relationship between changes in iron metabolism-related factors in hypertrophic ligamentum flava tissue or cells, oxidative stress, and 
inflammation due to the iron metabolism imbalance. Therefore, the changes in the regulatory factors related to iron metabolism, homeostasis following activation of the fibrosis of ligamentum flava, and therapeutic effect of iron homeostasis regulation should be further examined.

\section{Conclusions}

Melittin can promote axon growth and enhance functional recovery by activating M2 macrophages via controlling macrophage iron metabolism in LSS rats.

\section{Abbreviations}

LSS, Lumbar spinal stenosis; H\&E, Hematoxylin and eosin; PBS, phosphate-buffered saline; PFA, paraformaldehyde; NGS, normal goat serum; FITC, fluorescein isothiocyanate; qPCR, real-time polymerase chain reaction; DRG, dorsal root ganglion; TRPV1, transient receptor potential vanilloid subtype 1; iNOS, inducible nitric oxide synthase; COX-2, cyclooxygenase-2; IL, interleukin, TNF-a, tumor necrosis factoralpha; Arg1, Arginase 1; Nrf2, nuclear factor erythroid 2-related factor 2; FTH1, ferritin heavy chain 1; HAMP, hepcidin antimicrobial peptide; DMT1, divalent metal transporter 1; TFRC, transferrin receptor; CP, ceruloplasmin; NF200, neurofilament 200; ANOVA, one-way analysis of variance.

\section{Declarations}

\section{Ethics approval and consent to participate}

Ethics approval: All procedures were approved by the Jaseng Animal Care and Use Committee (JSR-201909-022-001).

Consent to participate: Not applicable.

\section{Consent for publication}

Not applicable.

\section{Availability of data and materials}

The datasets used and/or analyzed during the current study are available from the corresponding author on reasonable request.

\section{Competing interests}

The author declare that they have no competing interests.

\section{Funding}

This research was funded by the Jaseng Medical Foundation, Korea. Institutional 


\section{Author contributions}

Conceptualization, H.K.; methodology, H.K. and J.Y.H.; software, H.K.; validation, H.K., J.Y.H., J.L. and W.J.J.; formal analysis, H.K., J.Y.H., J.L. and W.-J.J.; investigation, H.K.; resources, I.-H.H.; data curation, H.K.; writing-original draft preparation, H.K.; writing - review and editing, Y.J.L. and I.-H.H.; visualization, H.K. and J.L.; supervision, I.-H.H.; project administration, I.-H.H.; funding acquisition, I.-H.H.

All authors read and approved the final manuscript

\section{Acknowledgements}

This work was supported by a grant from the Traditional Korea Medicine Research and Development Program of the Korean Health Industry Development Institute (KHIDI), funded by the Ministry of Health and Welfare, Republic of Korea (grant number: HF21C0100)

\section{Author information}

Affiliations: Jaseng Spine and Joint Research Institute, Jaseng Medical Foundation, Seoul 135-896, Republic of Korea.

\section{References}

[1] N. Fujita, A. Sakurai, A. Miyamoto, T. Michikawa, O. Tsuji, N. Nagoshi, E. Okada, M. Yagi, Y. Otaka, T. Tsuji, H. Kono, K. Ishii, M. Nakamura, M. Matsumoto, K. Watanabe, Lumbar spinal canal stenosis leads to locomotive syndrome in elderly patients, J Orthop Sci 24(1) (2019) 19-23.

[2] G.C. Brown, Living too long: the current focus of medical research on increasing the quantity, rather than the quality, of life is damaging our health and harming the economy, EMBO Rep 16(2) (2015) 13741.

[3] S. Genevay, S.J. Atlas, Lumbar spinal stenosis, Best Pract Res Clin Rheumatol 24(2) (2010) 253-65.

[4] B.H. Lee, S.H. Moon, K.S. Suk, H.S. Kim, J.H. Yang, H.M. Lee, Lumbar Spinal Stenosis: Pathophysiology and Treatment Principle: A Narrative Review, Asian Spine J 14(5) (2020) 682-693.

[5] J. Chen, Z. Liu, G. Zhong, L. Qian, Z. Li, Z. Qiao, B. Chen, H. Wang, Hypertrophy of ligamentum flavum in lumbar spine stenosis is associated with increased miR-155 level, Dis Markers 2014 (2014) 786543.

[6] P.W.H. Cheung, V. Tam, V.Y.L. Leung, D. Samartzis, K.M. Cheung, K.D. Luk, J.P.Y. Cheung, The paradoxical relationship between ligamentum flavum hypertrophy and developmental lumbar spinal stenosis, Scoliosis Spinal Disord 11(1) (2016) 26.

[7] K. Sairyo, A. Biyani, V. Goel, D. Leaman, R. Booth, Jr., J. Thomas, D. Gehling, L. Vishnubhotla, R. Long, N. Ebraheim, Pathomechanism of ligamentum flavum hypertrophy: a multidisciplinary investigation 
based on clinical, biomechanical, histologic, and biologic assessments, Spine (Phila Pa 1976) 30(23) (2005) 2649-56.

[8] H.J. Moon, Y.K. Park, Y. Ryu, J.H. Kim, T.H. Kwon, H.S. Chung, J.H. Kim, The angiogenic capacity from ligamentum flavum subsequent to inflammation: a critical component of the pathomechanism of hypertrophy, Spine (Phila Pa 1976) 37(3) (2012) E147-55.

[9] J.B. Park, H. Chang, J.K. Lee, Quantitative analysis of transforming growth factor-beta 1 in ligamentum flavum of lumbar spinal stenosis and disc herniation, Spine (Phila Pa 1976) 26(21) (2001) E492-5.

[10] K. Sairyo, A. Biyani, V.K. Goel, D.W. Leaman, R. Booth, Jr., J. Thomas, N.A. Ebraheim, I.A. Cowgill, S.E. Mohan, Lumbar ligamentum flavum hypertrophy is due to accumulation of inflammation-related scar tissue, Spine (Phila Pa 1976) 32(11) (2007) E340-7.

[11] S.J. Atlas, A. Delitto, Spinal stenosis: surgical versus nonsurgical treatment, Clin Orthop Relat Res 443 (2006) 198-207.

[12] W.T. Enthoven, P.D. Roelofs, R.A. Deyo, M.W. van Tulder, B.W. Koes, Non-steroidal anti-inflammatory drugs for chronic low back pain, Cochrane Database Syst Rev 2 (2016) CD012087.

[13] P. Handa, S. Thomas, V. Morgan-Stevenson, B.D. Maliken, E. Gochanour, S. Boukhar, M.M. Yeh, K.V. Kowdley, Iron alters macrophage polarization status and leads to steatohepatitis and fibrogenesis, J Leukoc Biol 105(5) (2019) 1015-1026.

[14] A. Kroner, A.D. Greenhalgh, J.G. Zarruk, R. Passos Dos Santos, M. Gaestel, S. David, TNF and increased intracellular iron alter macrophage polarization to a detrimental M1 phenotype in the injured spinal cord, Neuron 83(5) (2014) 1098-116.

[15] R. Gozzelino, P. Arosio, Iron Homeostasis in Health and Disease, Int J Mol Sci 17(1) (2016).

[16] C. Kaindlstorfer, K.A. Jellinger, S. Eschlbock, N. Stefanova, G. Weiss, G.K. Wenning, The Relevance of Iron in the Pathogenesis of Multiple System Atrophy: A Viewpoint, J Alzheimers Dis 61(4) (2018) 12531273 .

[17] M. Carpena, B. Nunez-Estevez, A. Soria-Lopez, J. Simal-Gandara, Bee Venom: An Updating Review of Its Bioactive Molecules and Its Health Applications, Nutrients 12(11) (2020).

[18] G. Lee, H. Bae, Anti-Inflammatory Applications of Melittin, a Major Component of Bee Venom: Detailed Mechanism of Action and Adverse Effects, Molecules 21(5) (2016).

[19] H. Kim, J.Y. Hong, W.J. Jeon, S.H. Baek, I.H. Ha, Bee Venom Melittin Protects against CisplatinInduced Acute Kidney Injury in Mice via the Regulation of M2 Macrophage Activation, Toxins (Basel) 12(9) (2020). 
[20] C. Lee, S.S. Bae, H. Joo, H. Bae, Melittin suppresses tumor progression by regulating tumorassociated macrophages in a Lewis lung carcinoma mouse model, Oncotarget 8(33) (2017) 5495154965.

[21] S. Faissner, M. Mishra, D.K. Kaushik, J. Wang, Y. Fan, C. Silva, G. Rauw, L. Metz, M. Koch, V.W. Yong, Systematic screening of generic drugs for progressive multiple sclerosis identifies clomipramine as a promising therapeutic, Nat Commun 8(1) (2017) 1990.

[22] S. Recalcati, M. Locati, E. Gammella, P. Invernizzi, G. Cairo, Iron levels in polarized macrophages: regulation of immunity and autoimmunity, Autoimmun Rev 11(12) (2012) 883-9.

[23] C. Gaetano, L. Massimo, M. Alberto, Control of iron homeostasis as a key component of macrophage polarization, Haematologica 95(11) (2010) 1801-3.

[24] X. Chen, C. Guo, J. Kong, Oxidative stress in neurodegenerative diseases, Neural Regen Res 7(5) (2012) 376-85.

[25] G.H. Kim, J.E. Kim, S.J. Rhie, S. Yoon, The Role of Oxidative Stress in Neurodegenerative Diseases, Exp Neurobiol 24(4) (2015) 325-40.

[26] B. Poljsak, D. Suput, I. Milisav, Achieving the balance between ROS and antioxidants: when to use the synthetic antioxidants, Oxid Med Cell Longev 2013 (2013) 956792.

[27] R.J. Ward, F.A. Zucca, J.H. Duyn, R.R. Crichton, L. Zecca, The role of iron in brain ageing and neurodegenerative disorders, Lancet Neurol 13(10) (2014) 1045-60.

[28] J. Xu, M.D. Knutson, C.S. Carter, C. Leeuwenburgh, Iron accumulation with age, oxidative stress and functional decline, PLoS One 3(8) (2008) e2865.

\section{Tables}

Table 1 is not available with this version

\section{Figures}



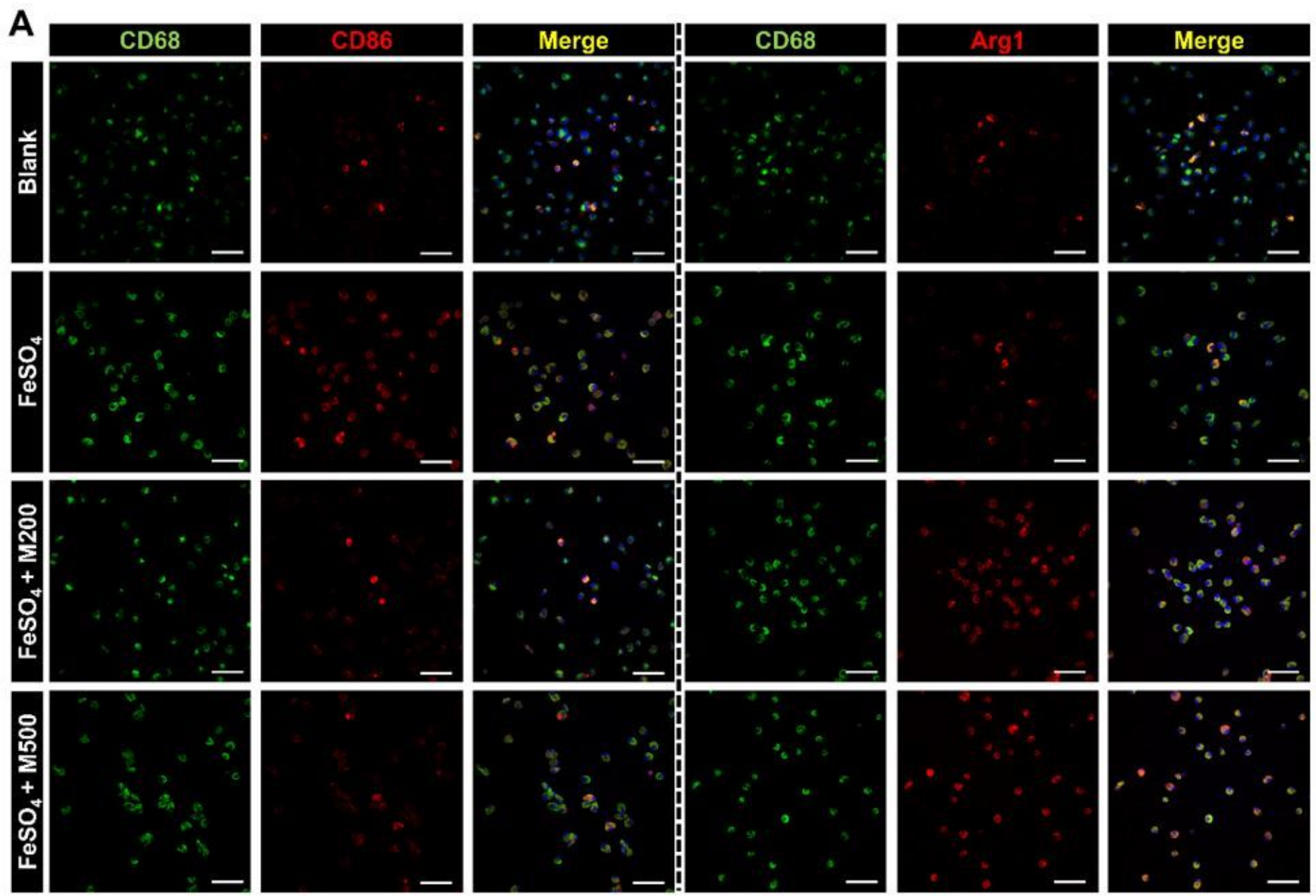

B

M1 type

C
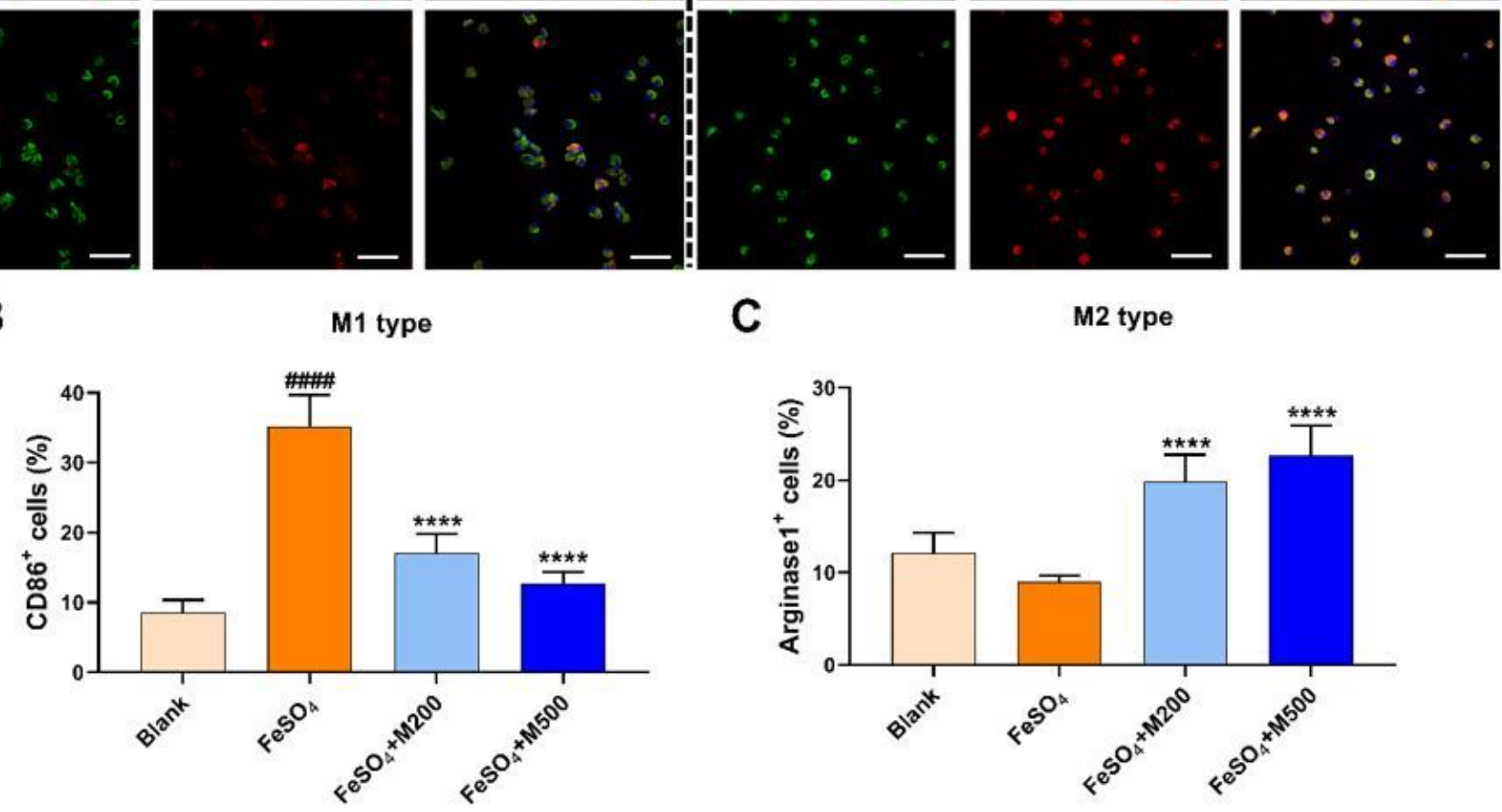

M2 type

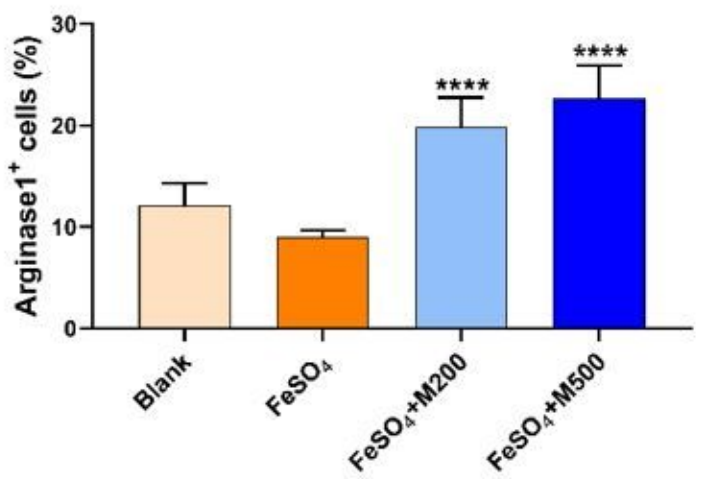

Figure 1

Melittin inhibits iron accumulation via the regulation of macrophage phenotype. A) Representative images of M1 macrophages (CD86 positive, double-stained with ED1) and M2 macrophages (Arginase 1 positive, double-stained with ED1). White scale bar $=50 \mu \mathrm{m}$. (B) Quantitative percentage of the CD86stained cells (M1) in total CD68-positive cells. (C) Quantitative percentage of the Arg1-stained cells (M2) in total CD68-positive cells. Data are expressed as the means \pm SEM. Significant differences indicated as 
$\# \# \# p<0.0001$ vs. the blank group and ${ }^{* \star \star \star} \mathrm{p}<0.0001$ vs. the $\mathrm{FeSO}_{4}$-treated group were analyzed via oneway ANOVA with Tukey's post-hoc test.

A
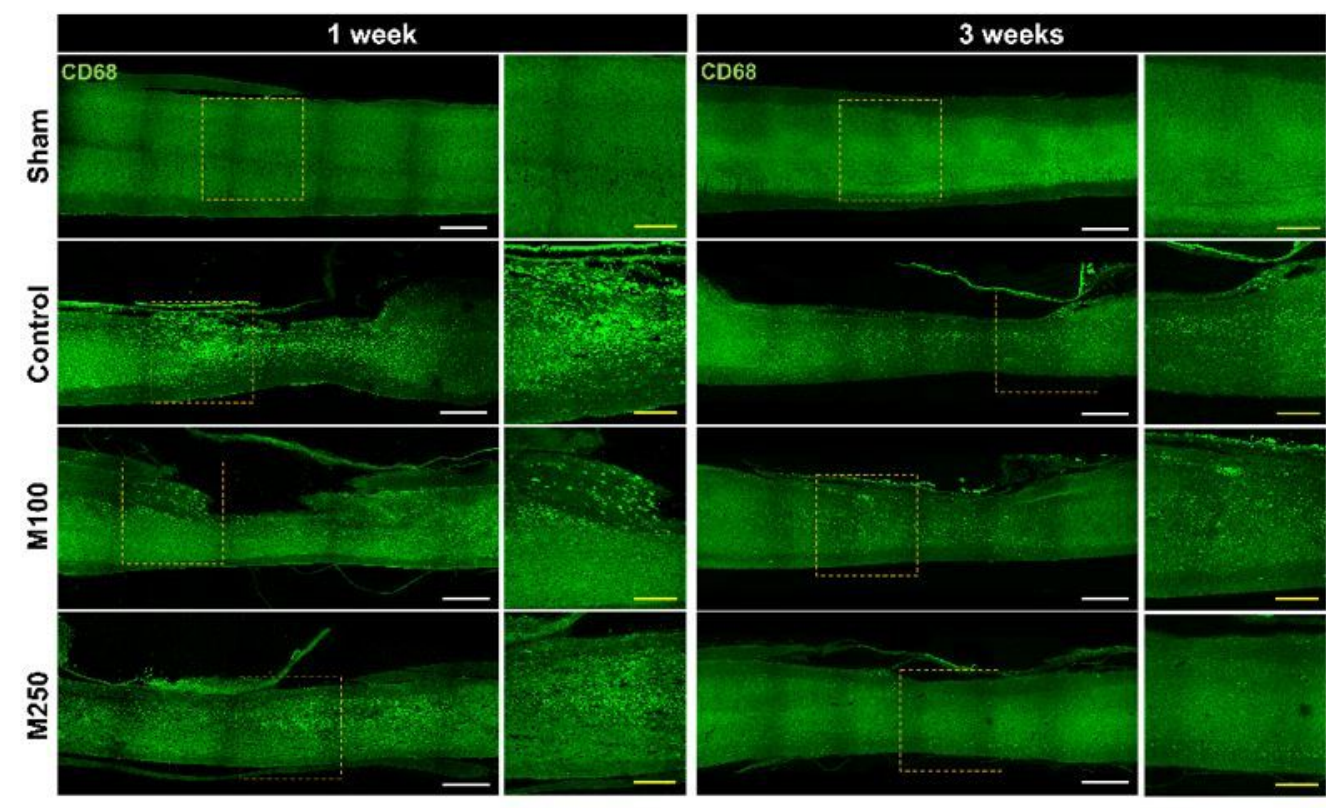

E
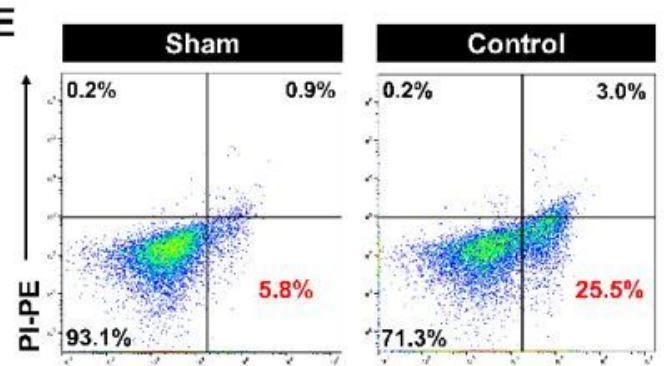

Annexin V-FITC
B

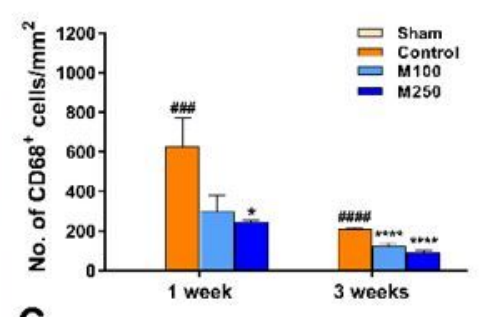

C

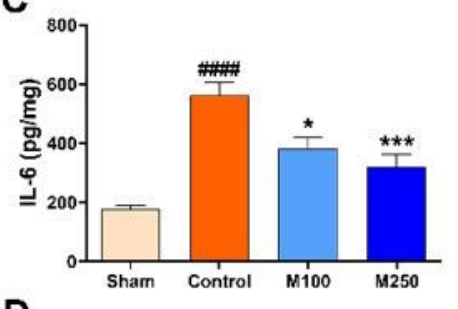

D

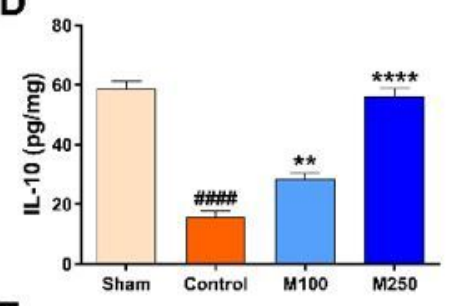

$\mathbf{F}$

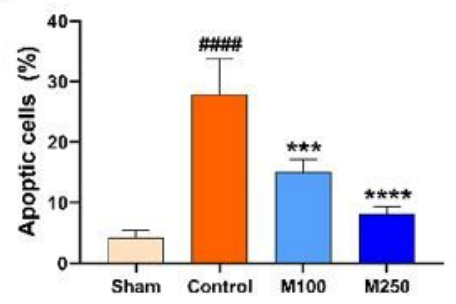

Figure 2

Melittin inhibits inflammation responses in LSS rats. (A) Representative images of immunohistochemical analysis of CD68 (green) in the injured spinal cord. White scale bar $=50 \mu \mathrm{m}$. (B) Quantitative number of CD86-stained cells (M1) in each group. (C, D) ELISA analysis of (C) IL-6 and (D) IL-10 in isolated spinal cord in each group. (E) Early and late apoptosis in spinal cord cells. (F) Percentage of apoptotic cells in each group. Data are expressed as the means \pm SEM. Significant differences are indicated as ${ }^{\# \# \#} \mathrm{p}<$

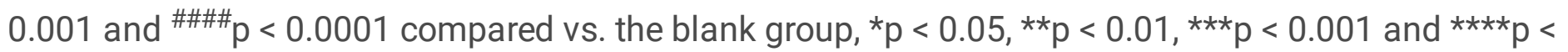
0.0001 vs. the control group were analyzed via one-way ANOVA with Tukey's post-hoc test. 
A

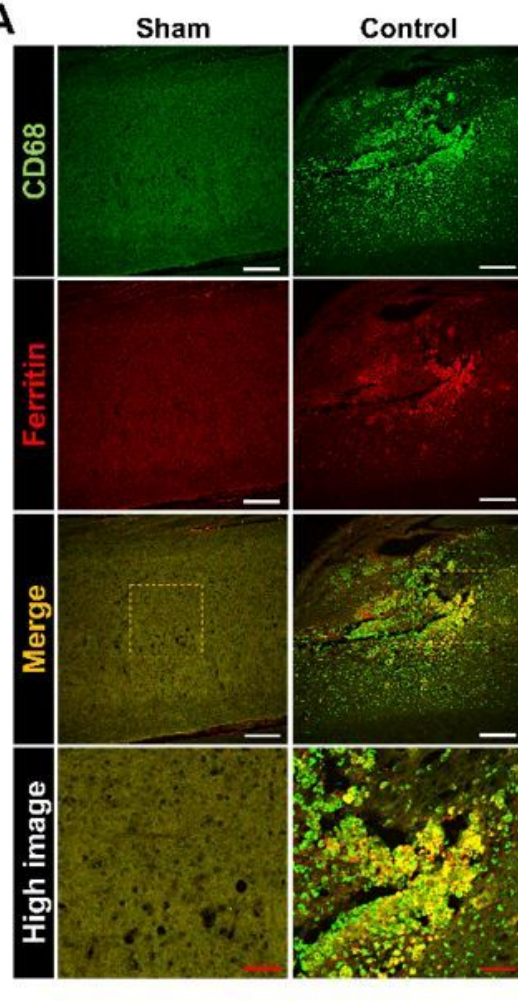

M100
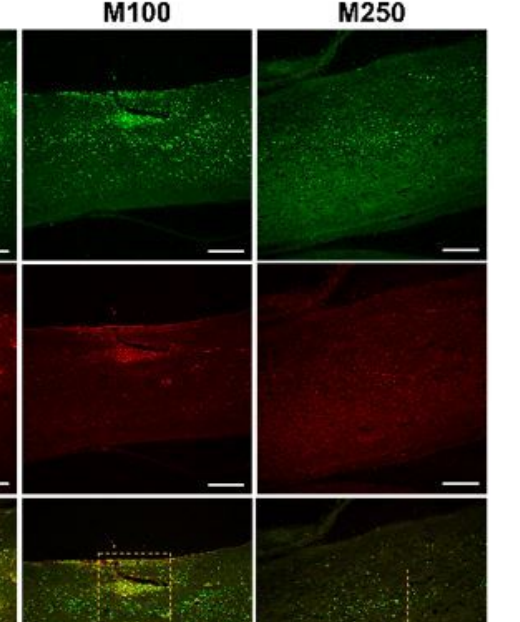

S.

atinc....
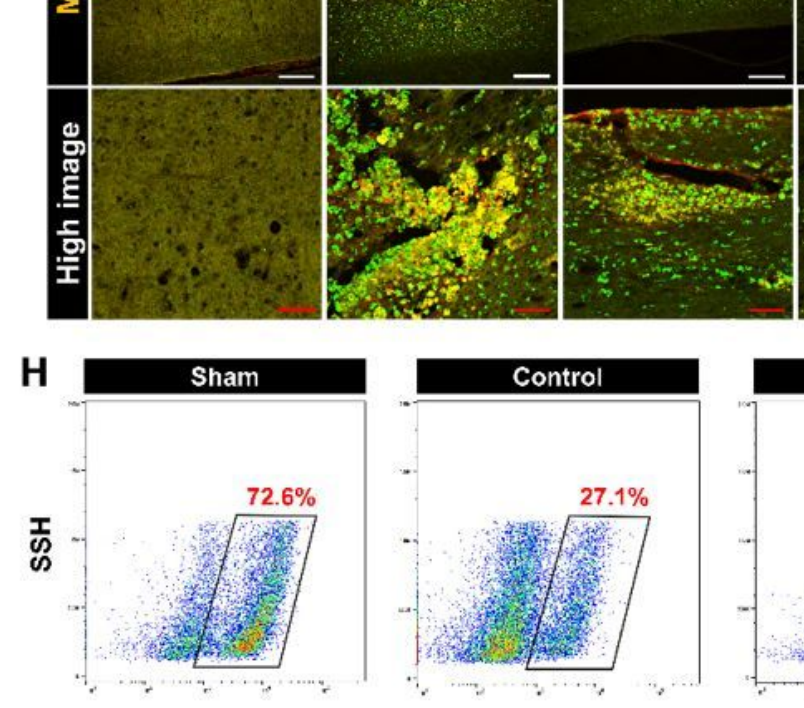

B
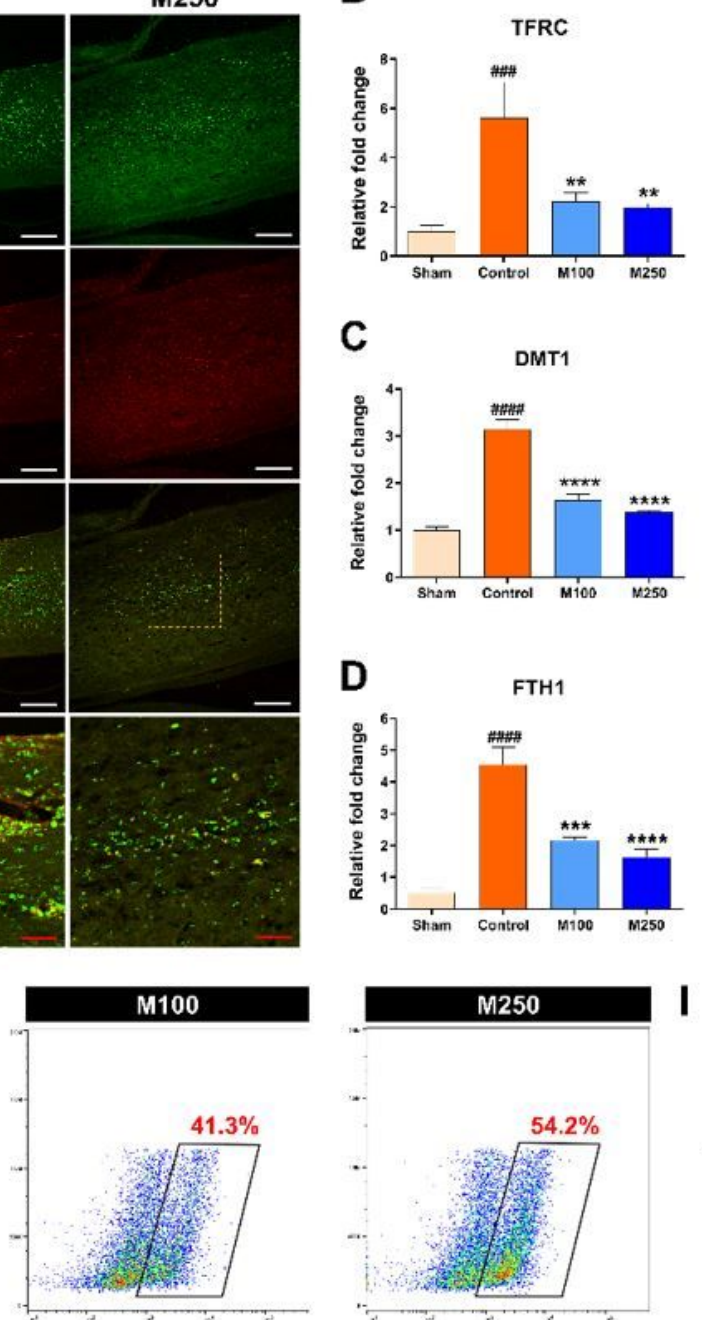

E

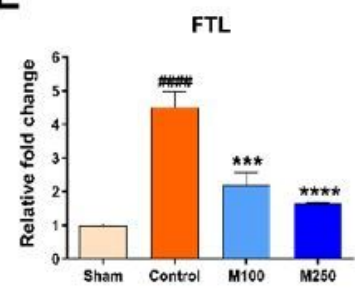

F
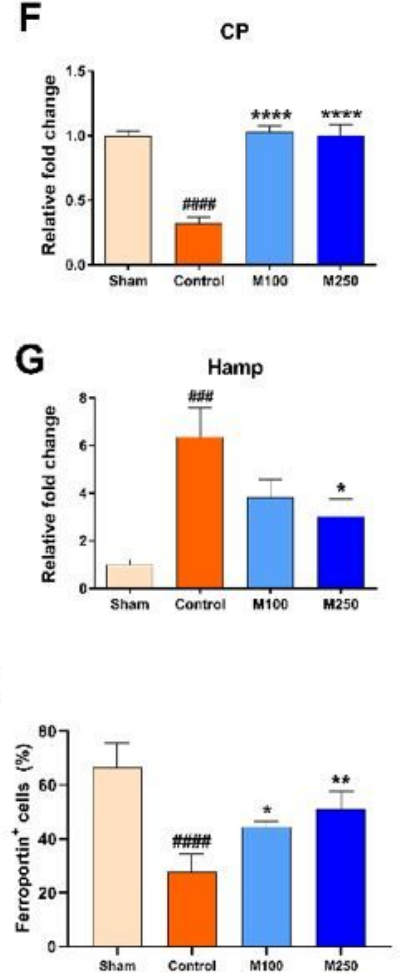

Ferroportin-PE

\section{Figure 3}

Melittin inhibits inflammation responses in LSS rats. (A) Representative images of immunohistochemical analysis of CD68 (green) in the injured spinal cord. White scale bar $=50 \mu \mathrm{m}$. (B) Quantitative number of CD86-stained cells (M1) in each group. (C, D) ELISA analysis of (C) IL-6 and (D) IL-10 in isolated spinal cord in each group. (E) Early and late apoptosis in spinal cord cells. (F) Percentage of apoptotic cells in each group. Data are expressed as the means \pm SEM. Significant differences are indicated as ${ }^{\# \# \# p<}$

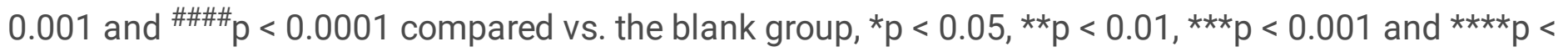
0.0001 vs. the control group were analyzed via one-way ANOVA with Tukey's post-hoc test. 

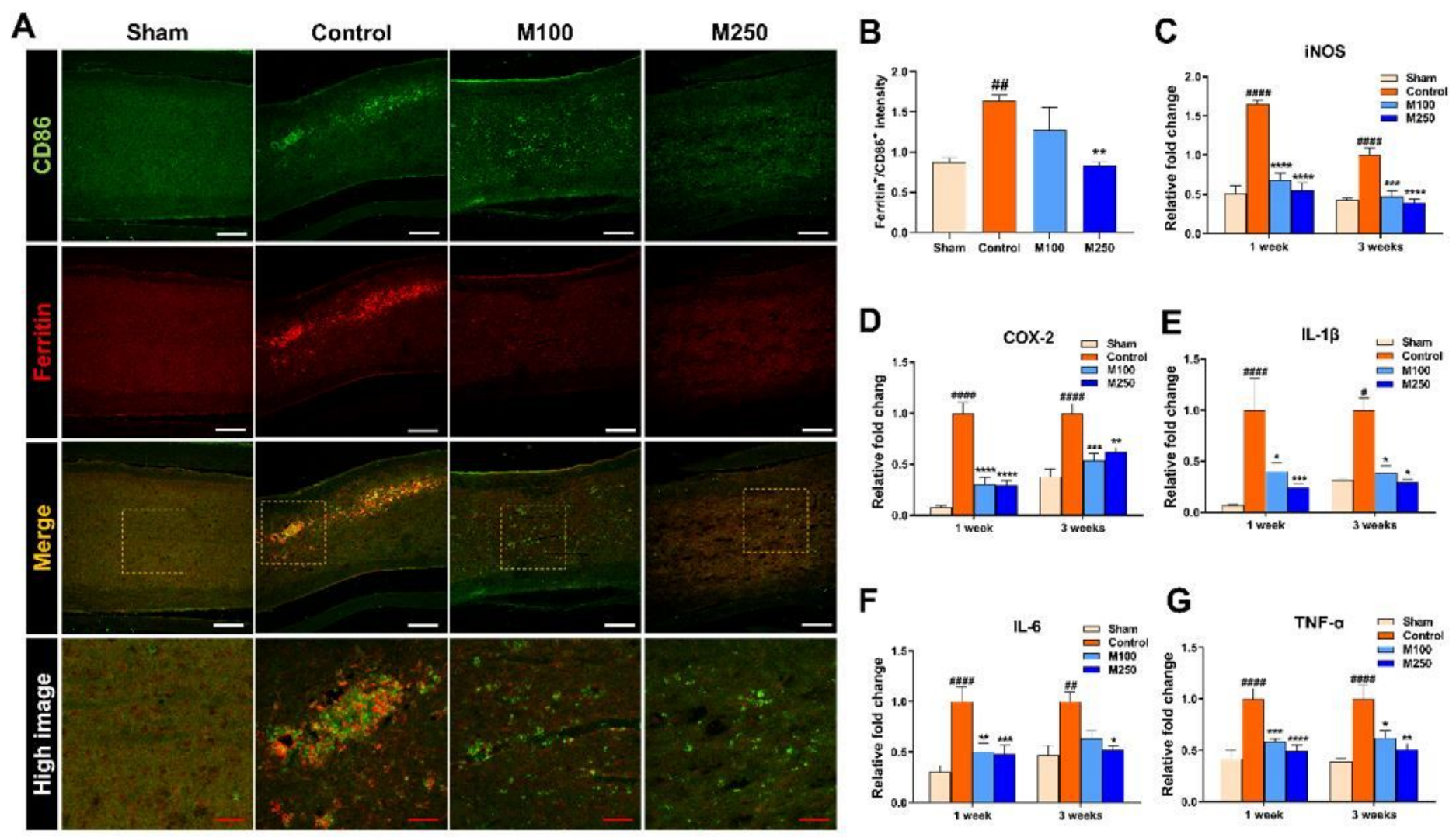

Figure 4

Melittin alleviates inflammation through inhibiting iron accumulation within the M1 macrophage phenotype. (A) Representative immunohistochemical images of M1 macrophages positive for CD86 (green) and ferritin (red) in the spinal cord of LSS rats. (B) The percentage of cells that express both ferritin and CD86 in the spinal cords of LSS rats at 1 week. Quantification data from real time PCR for inflammation-related genes (C) iNOS, (D) COX-2, (E) IL-1 $\beta$, (F) IL-6, (G) TNF- $a$ at 1 and 3 weeks in LSS rats. Data are expressed as the means \pm SEM. Significant differences are indicated as ${ }^{\# \#} p<0.01$ and ${ }^{\# \# \# p<}$ 0.0001 compared vs. the sham group, ${ }^{*} p<0.05$, ${ }^{*} p<0.01$, ${ }^{\star *} \mathrm{p} p<0.001$ and ${ }^{* \star * \star} p<0.0001$ vs. the control group were analyzed via one-way ANOVA with Tukey's post-hoc test. 
A
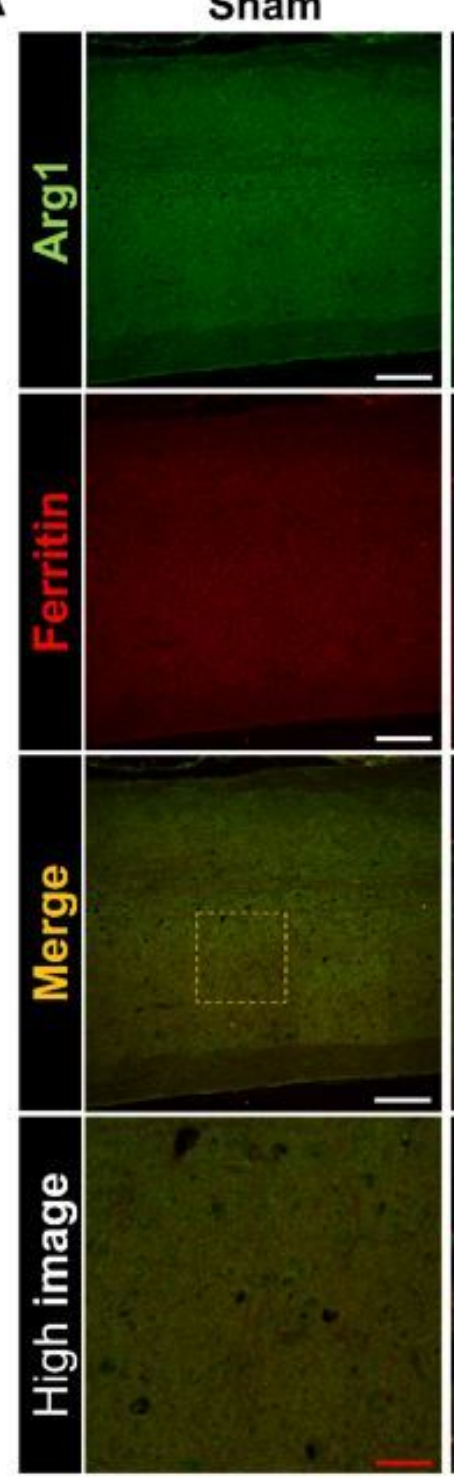

B

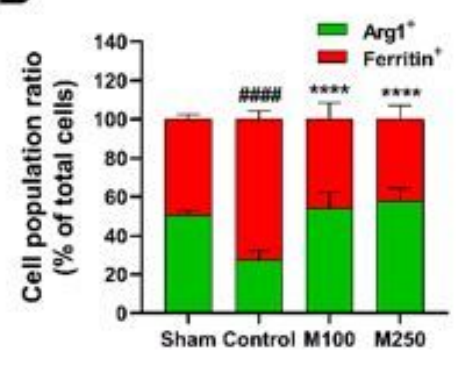

Control

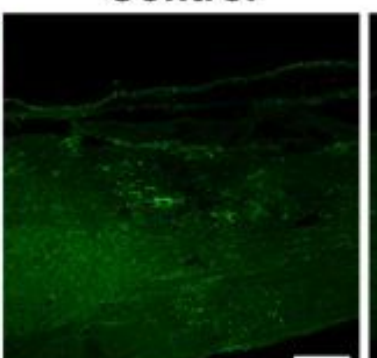

M100
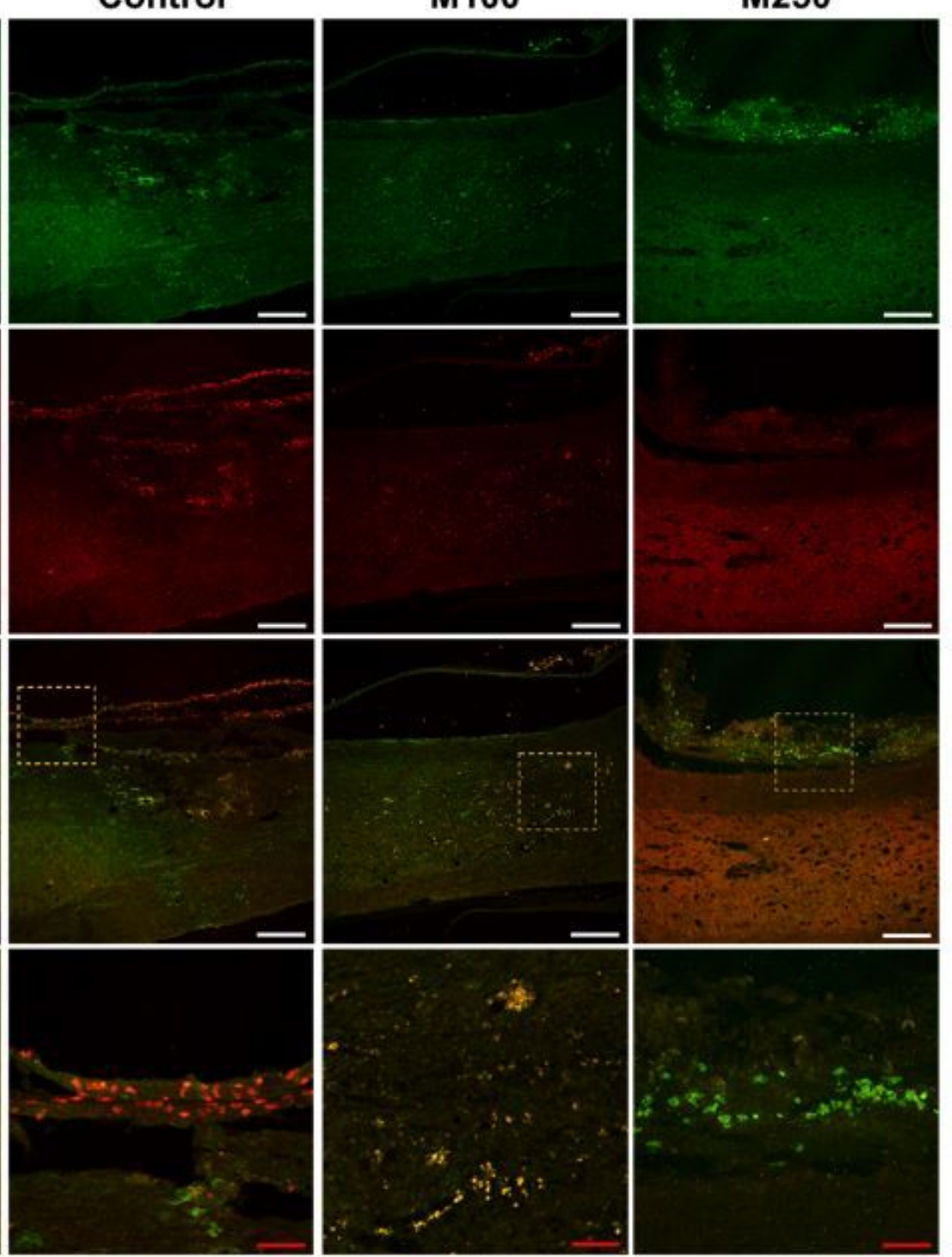

C

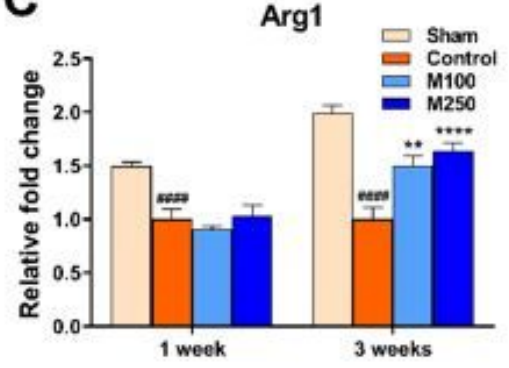

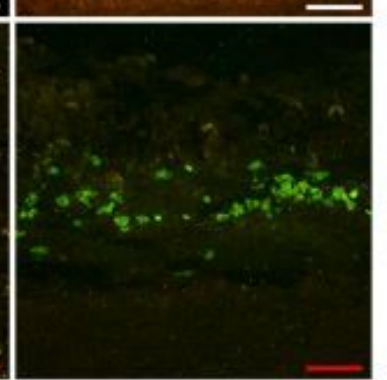

M250

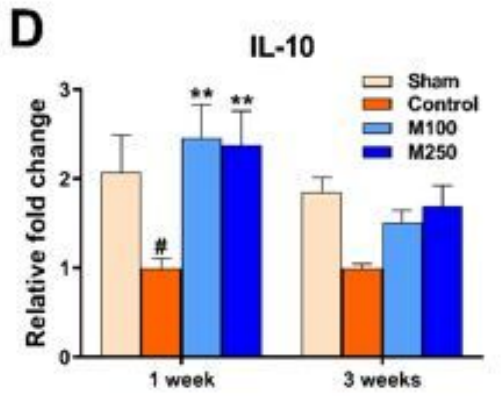

\section{Figure 5}

Melittin promotes M2 macrophage polarization with inhibition of iron deposition. (A) Representative immunohistochemical images of M2 macrophages positive for Arg1 (green) and ferritin (red) in the spinal cord of LSS rats. White scale bar $=200 \mu \mathrm{m}$. Red scale bar $=50 \mu \mathrm{m}$. (B) The percentage of cells that express both ferritin and Arg1 in spinal cord of LSS rats at 1 week. Quantification data from real time PCR for genes related to anti-inflammatory pathway (C) $\operatorname{Arg} 1,(\mathrm{D}) / L-10$ at 1 and 3 weeks in LSS rats. Data are 
expressed as the means \pm SEM. Significant differences are indicated as $\#_{p}<0.01$ and ${ }^{\# \# \# p<0.0001}$ compared vs. the sham group, ${ }^{\star *} p<0.01$ and ${ }^{\star \star \star *} p<0.0001$ vs. the control group were analyzed via oneway ANOVA with Tukey's post-hoc test.

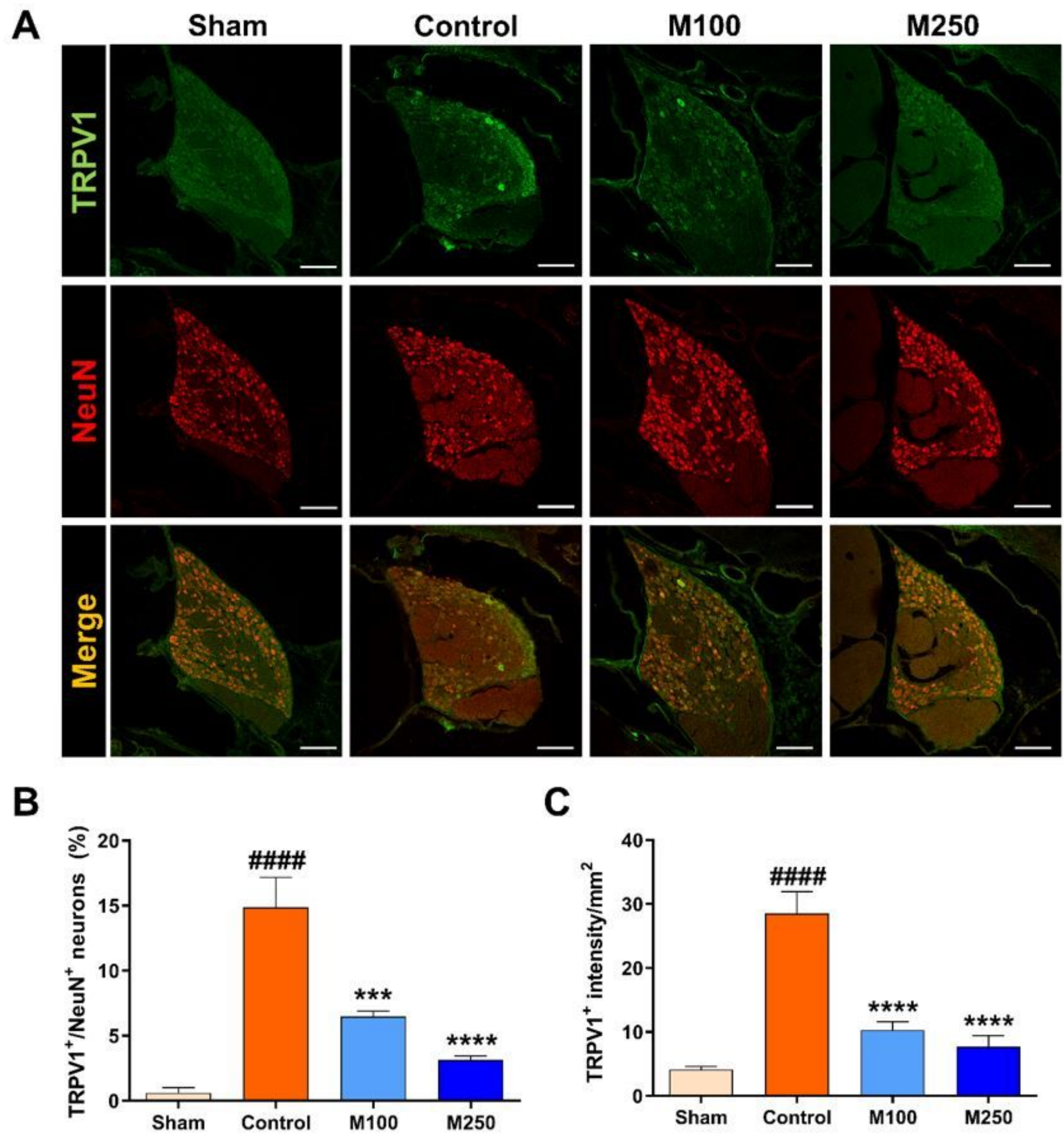

Figure 6 
Melittin relieves neuropathic pain through TRPV1 inhibition in LSS rats. (A) Representative immunohistochemical images from each group of TRPV1 (green) and NeuN (red) in the DRG at 3 weeks after LSS. White scale bar $=200 \mu \mathrm{m}$. (B) Percentage of co-labeled neurons with TRPV1 and NeuN in DRG. (C) Quantification of fluorescence intensity in TRPV1-positive neurons within DRG. Data are expressed as

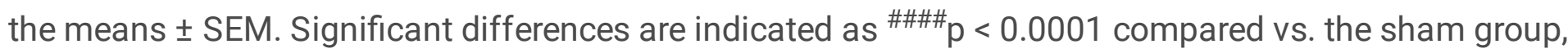
$\star \star \star x p<0.001$ and $* \star \star \star p p<0.0001$ vs. the control group were analyzed via one-way ANOVA with Tukey's post-hoc test.

A
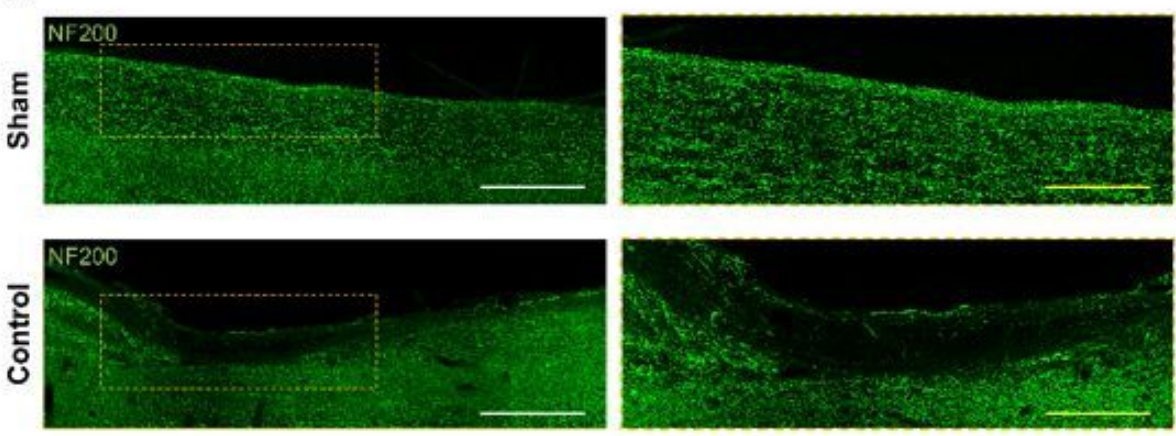

B
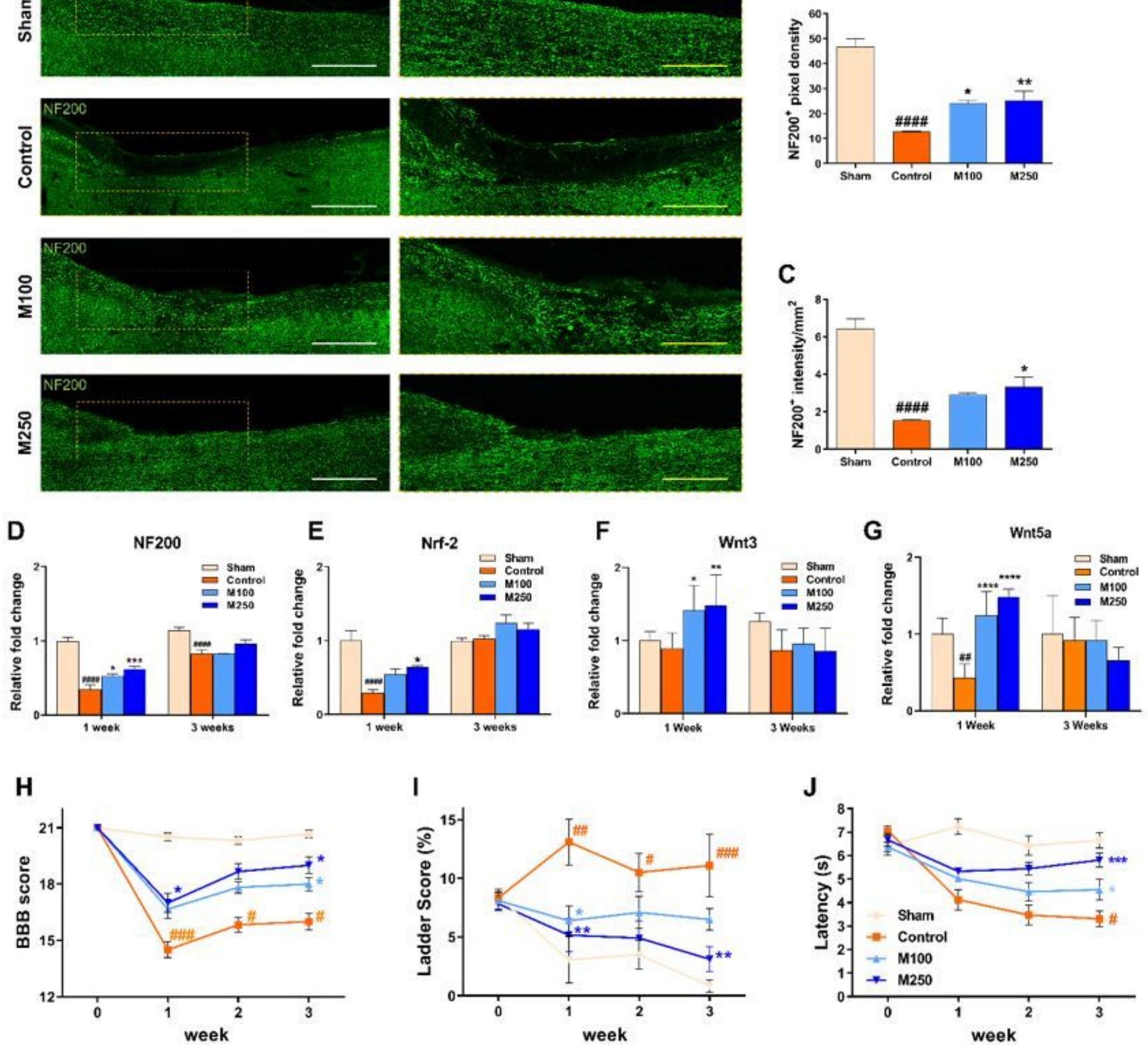

Figure 7 
Melittin promotes axonal growth and enhances locomotor recovery in LSS rats. (A) Representative immunohistochemical images of NF200 (green) in spinal cord at 3 weeks after LSS from each group. The pixel density (B) and intensity (C) of NF200-positive axons of each group. Real time PCR analysis of mRNA expression levels of regeneration-related genes, (D) NF200, (E) Nrf2, (F) Wnt3 and (F) Wnt5a at 1 and 3 weeks in each group. Three locomotor assessments, $(\mathrm{H})$ BBB test, $(\mathrm{I})$ Ladder test, and $(\mathrm{J})$ Von Frey test, of the LSS rats administered with melittin until 3 weeks. Data are expressed as the means \pm SEM.

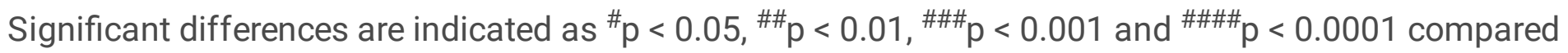
vs. the sham group, ${ }^{\star} p<0.05,{ }^{\star \star} p<0.01,{ }^{\star \star \star} p<0.001$ and ${ }^{\star \star \star \star} p<<0.0001$ vs. the control group were analyzed via one-way ANOVA with Tukey's post-hoc test.

\section{Supplementary Files}

This is a list of supplementary files associated with this preprint. Click to download.

- GraphicalAbstract.docx 